\title{
EDAD, TRABAJO Y SALUD OCUPACIONAL: RETOS Y OPORTUNIDADES PARA LOS TRABAJADORES Y LAS ORGANIZACIONES EN IBEROAMÉRICA
}

\author{
AGE, WORK AND OCCUPATIONAL HEALTH: \\ CHALLENGES AND OPPORTUNITIES FOR WORKERS AND \\ ORG ANIZATIONS IN IBERO-AMERICA
}

\author{
CARLOS-MARÍA ALCOVER \\ Universidad Rey Juan Carlos, España \\ *Autor de correspondencia: \\ carlosmaria.alcoveraurjc.es
}

El envejecimiento de la fuerza de trabajo debido a los cambios sociodemográficos globales plantea un conjunto de retos y de oportunidades cuya gestión es compleja y cuyos resultados pueden traer consigo consecuencias muy diversas para las personas y para las organizaciones. El principal objetivo de este artículo es analizar los más importantes identificados por la investigación internacional aplicados al contexto iberoamericano. Tras una contextualización de esos cambios sociodemográficos y sus impactos en el mundo del trabajo en los países de Iberoamérica, se abordan tres de los principales retos. En primer lugar, el relacionado con los conceptos de edad cronológica y edad subjetiva, así como otros tipos de conceptualizaciones de la edad aplicados en contextos organizacionales y laborales. En segundo lugar, analizar la evidencia disponible sobre la motivación, las actitudes y el desempeño de los trabajadores mayores, con objeto de reflexionar y cuestionar los estereotipos heredados acerca de su comportamiento y capacidad laboral. Y en tercer lugar, identificar los principales elementos del enfoque de la empleabilidad sostenible, con el que se pretende responder al reto de mantener y prolongar la vida laboral de los trabajadores mayores en buenas condiciones de satisfacción, desempeño, bienestar y salud. El artículo concluye identificando algunas de las principales futuras líneas de investigación e intervención en este ámbito cuya importancia seguirá aumentando en los próximos años

Palabras claves: envejecimiento, trabajadores mayores, gestión de la edad, salud ocupacional, empleabilidad sostenible.
The aging of the workforce due to global socio-demographic changes poses a set of challenges and opportunities that are complex to manage and whose results can have diverse consequences for individuals and organizations. The main objective of this article is to analyze the most important ones identified by international research applied to the IberoAmerican context. After a contextualization of these sociodemographic changes and their impact on the world of work in the countries of the region, three of the main challenges are addressed. Firstly, that related to the concepts of chronological age and subjective age, as well as other types of conceptualizations of age applied in organizational and labor contexts. Secondly, to analyze the available evidence on the motivation, attitudes, and performance of older workers, to reflect on and question inherited stereotypes about their work ability and behavior. And thirdly, to identify the main elements of the sustainable employability approach, which aims to respond to the challenge of maintaining and prolonging the working lives of older workers under ideal conditions of satisfaction, performance, well-being, and health. The article concludes by identifying some of the main future lines of research and intervention in this field, the importance of which will continue to grow in the coming years.

Keywords:ging, older workers, age management, occupational health, sustainable employability. 


\section{INTRODUCCIÓN}

El actual envejecimiento de la población mundial tiene importantes consecuencias para los sistemas de pensiones y de protección social, la salud, la educación, el trabajo, la cohesión social e intergeneracional, la vivienda o el acceso a los recursos materiales y tecnológicos necesarios para garantizar una vida autónoma y digna en la vejez. En este contexto de envejecimiento generalizado, las políticas y prácticas que facilitan el envejecimiento activo resultan prioritarias (Fernández et al., 2018; Kooij et al., 2020; Troncoso Pantoja et al., 2020). Si bien en el proceso de envejecimiento juegan un papel fundamental otras tareas y experiencias vitales y los roles no determinados económicamente (Moulaert $\&$ Biggs, 2013), uno de los ejes que posibilitan el envejecimiento activo es la actividad laboral, puesto que constituye uno de los ámbitos con mayores implicaciones para el mantenimiento de la actividad física y mental, de las relaciones sociales, de los ingresos económicos básicos, de la integración en la sociedad, de las identidades personal y sociales y de una vida significativa (Osborne \& Newman, 2012). Sin embargo, la continuidad de los adultos mayores en el mercado laboral representa afrontar simultáneamente un conjunto de retos y de oportunidades cuya gestión es compleja y cuyos resultados pueden traer consigo consecuencias muy diversas para las personas y para las organizaciones.

El principal objetivo de este artículo es analizar los retos y oportunidades más importantes identificados por la investigación internacional aplicados al contexto iberoamericano. Tras una contextualización de los cambios sociodemográficos y sus impactos en el mundo del trabajo en los países de Iberoamérica, se abordan tres de los principales retos. En primer lugar, el relacionado con los conceptos de edad cronológica y edad subjetiva, así como otros tipos de conceptualizaciones de la edad aplicados en contextos organizacionales y laborales. En segundo lugar, analizar la evidencia disponible sobre la motivación, las actitudes y el desempeño de los trabajadores mayores, con objeto de cambiar los estereotipos heredados acerca de su comportamiento laboral. Y en tercer lugar, identificar los principales elementos del enfoque de la empleabilidad sostenible, un constructo que puede contribuir a responder al reto de mantener y prolongar la vida laboral de los trabajadores mayores en buenas condiciones de desempeño, satisfacción, bienestar y salud. El artículo concluye identificando algunas de las principales futuras líneas de investigación e intervención.

\section{CONTEXTUALIZACIÓN DE LOS CAMBIOS SOCIODE- MOGRÁFICOS Y LABORALES EN IBEROAMÉRICA}

En términos globales, de los 7713 millones de personas que vivían en el mundo en 2019, en 2030 se estima que se pasará a los 8548 millones y en 2050, a los 9735 millones. En lo que respecta a los países de Iberoamérica lque incluye la región de América Latina, incluido Brasil, y el Caribe), la progresión estimada será de 648 en 2019 a 706 y 762 millones en esos mismos años. En cuanto al porcentaje de población de más de 65 años, las estimaciones también señalan importantes incrementos. En el mundo, en 2019 el 9,1\% ya formaba parte de ese grupo de edad, y se estima que en 2030 será el $11,7 \%$ y en 2050, el 15,9\%. En América Latina y el Caribe, en 2019 la población mayor de 65 años representaba un $8,7 \%$, en 2030 se espera que alcance el $12 \%$, y en 2050 llegará al 19\% (United Nations, 2019). Como puede comprobarse, en solo 30 años el número de personas del grupo de 
más edad se doblará y el incremento en esta región será mucho mayor que en el global del mundo, superándolo en más de 3 puntos, datos que muestran no solo el volumen sino también el acelerado proceso de envejecimiento. Otro indicador significativo de ello es el rápido aumento de la esperanza de vida al nacer; en 2020, en los países de América del Sur era de 80 años para las mujeres y 72 para los hombres; en América Central y México, de 78 y 72 años, respectivamente; y en El Caribe, de 75 y 71 años. En conjunto, para toda la región, la esperanza de vida al nacer en 2020 era de 79 años para las mujeres y 72 para los hombres (Population Reference Bureau, 2021), y en 2050 llegará a en conjunto a los 80,9 años 183,2 para las mujeres y 78,5 para los hombres; United Nations, 2019). En general, como puede comprobarse a partir de los datos anteriores, en solo 30 años se producirá un significativo incremento en Iberoamérica, reduciéndose además la brecha de años entre los géneros. Todos estos indicadores muestran que los países de la región dispondrán de menos tiempo que los de otras, como Europa o América del Norte, para adaptarse a las consecuencias del envejecimiento (Berganza et al., 2020). No obstante, el efecto de la pandemia de la COVID-19 ya ha mostrado descensos de más de un año de esperanza de vida al nacer, tanto en hombres como en mujeres, en países como Chile, similares a los comprobados en Estados Unidos y en los países de la Unión Europea (Aburto et al., 2021).

Este escenario demográfico tiene importantes repercusiones en el mundo del trabajo y en las sociedades en su conjunto. Por una parte, los aumentos combinados de la esperanza de vida y la tasa de dependencia Inúmero de personas en edad dependiente por cada persona en edad de trabajar) obligan a equilibrar los grupos de edad laboralmente activos, lo que supone mantener a los adultos mayores empleados y prolongar su vida laboral más allá de las edades de jubilación actuales (Alcover, 2020), utilizando, por ejemplo, modalidades de empleo puente (Alcover et al., 2014; Topa et al., 2014). Un efecto asociado del incremento de la esperanza de vida es la situación que afrontan cada vez más trabajadores de mediana edad y adultos mayores relativa a la obligación de cuidar a sus progenitores dependientes (Burch et al., 2019), tareas de cuidado que se pueden extender también a los hijos que aún viven en el domicilio familiar o a los nietos. A estos trabajadores que combinan su actividad laboral con las del doble cuidado familiar se les ha denominado como la Sandwich Generation (Burke, 2017), y tienen más probabilidad de cambiar a contratos a tiempo parcial, de sufrir evaluaciones negativas y discriminaciones en el trabajo, y de entrar en la jubilación anticipada a edades más tempranas (Converso et al., 2020; Henle et al., 2020), especialmente en el caso de las mujeres (Bauer \& Sousa-Poza, 2015), quienes siguen desempeñando mayoritariamente las tareas de cuidado.

Por otra parte, el número de años vividos en situación de jubilación continúa incrementándose, lo que ha llevado a algunos especialistas a diferenciar dos grupos de edad (Avdeev et al., 2011): por una parte, las personas comprendidas entre los 65 y los 80 años, y por otra, quienes superan los 80 y viven todavía un número variable de años, que puede ser aún elevado, puesto que la tasa de personas centenarias también sigue aumentando (Alcover, 2020). Este envejecimiento de las personas mayores también ha puesto de manifiesto la necesidad de modificar el uso de la edad cronológica como el único criterio para estimar el envejecimiento 
poblacional. En este sentido, se ha propuesto el uso de la edad prospectiva (Sanderson \& Scherbov, 2010), que indica la edad a la que se alcanza la "vejez" y deberían retirarse las personas cuando les falten 15 años de vida respecto a su esperanza de vida estimada. Un reciente estudio (Gietel-Basten et al., 2020) muestra que el rápido envejecimiento de la población en los países de América Central y el Caribe y en América del Sur es menos acentuado y más lento cuando se utiliza un umbral de vejez alternativo basado en la edad en la que la esperanza de vida restante es de 15 años, y se estima la tasa de dependencia de la vejez prospectiva en periodos de varias décadas. Este tipo de umbral de vejez (y de retirol ofrece una visión más precisa del envejecimiento real de los países que el basado únicamente en la edad cronológica (Gietel-Basten et al., 2020), y es más útil para que los gobiernos e instituciones adopten cambios y medidas tendentes a garantizar los sistemas de salud, las prestaciones sociales y las pensiones de las generaciones actuales y futuras. En los próximos años, el uso de modelos prospectivos y probabilísticos de envejecimiento de la población se acabará imponiendo sobre el modelo tradicional basado en la edad cronológica, y sus predicciones, además de parecer más realistas, apuntan a un envejecimiento menos intenso y acelerado que el pronosticado por el modelo tradicional (Sanderson et al., 2017).

Por último, la combinación de los factores señalados provoca que los actuales sistemas de pensiones y de protección social corran serios riesgos de colapsar, si bien las diferencias entre países hace que estos riesgos sean variables. Además, las grandes diferencias en los cambios demográficos y laborales y las enormes brechas de riqueza entre los núcleos urbanos de las grandes ciudades y los medios rurales, hacen que ni los problemas ni las soluciones sean únicos, una constante que caracteriza a América Latina y el Caribe, e incluso a los países de la Península Ibérica, desde hace décadas y que tiende a aumentar en el futuro próximo (Leeson, 2013). No obstante estas particularidades nacionales y geográficas, en muchos países de Iberoamérica (Boehs et al., 2017) las carreras laborales precarias y las bajas pensiones de jubilación provocan que muchos trabajadores no puedan jubilarse a las edades en que les corresponderían (Madero-Cabib et al., 2019), y les fuerzan a continuar trabajando, en ocasiones en unas malas condiciones de salud, tanto en el sector formal como, sobre todo, en el trabajo informal. Además, las desigualdades de género provocan que esta situación sea aún más negativa en el caso de las mujeres mayores (Vives et al., 2018). No obstante, cuando se analizan los macrodatos censales de los países de América Latina (de Souza et al., 2019) se encuentra que el estado de salud (la potencial mala salud de los adultos mayores) no es un obstáculo para su participación efectiva en el mercado de trabajo. Estos autores mostraron que a pesar de la mejora general de las condiciones de salud experimentada en los últimos años, este avance no se traduce en una mayor participación de los adultos mayores en la fuerza de trabajo en la actualidad con respecto al pasado. Así, aunque los trabajadores masculinos contemporáneos de 55-59 años tienen estados de salud similares a los de sus homólogos anteriores del grupo de edad de 60-64 años, sus patrones de participación en la fuerza de trabajo son muy diferentes (de Souza et al., 2019), lo que no se corresponde con la tendencia esperada de una mayor presencia en el mercado de trabajo. En principio, estos datos indicarían que el estado 
de salud no supondría una dificultad para aumentar las edades de jubilación en los países de América Latina, ni siquiera a largo plazo, según De Souza et al. (2019), sino que se trataría de otros factores estructurales y culturales, un debate político, social, sindical y económico siempre rodeado de fuertes controversias.

En definitiva, en estos escenarios de envejecimiento y de mercados de trabajo inestables y precarios (características que las consecuencias de la pandemia de la COVID-19 no han hecho sino acentuar desde 2019), los y las trabajadoras y las organizaciones se enfrentan a una serie de retos, pero también de oportunidades, relacionados con el envejecimiento en el trabajo y la salud ocupacional. A continuación se analizan los tres señalados al comienzo.

\section{RETO/OPORTUNIDAD 1: CAMBIAR LAS PERCEPCIONES SO- BRE LA EDAD Y EL ENVEJECIMIENTO EN EL TRABAJO}

Aunque desde hace décadas los expertos en ciclo vital han mostrado que el envejecimiento es un proceso multidimensional (p. ej., Baltes, 1987; Carstensen et al., 1999), que refleja cambios (que pueden implicar tanto ganancias como pérdidas) en el funcionamiento físico y fisiológico, cognitivo, afectivo, motivacional y social a lo largo del tiempo (Crawford et al., 2010; Kooij et al., 2008; Truxillo et al., 2015), la realidad es que laboral y socialmente continúa utilizándose la edad cronológica prácticamente como el único criterio para percibir, evaluar y tomar decisiones respecto a la carrera laboral de los adultos mayores. La dificultad encontrada para que los modelos y los datos de la investigación se trasladen a la sociedad y a las organizaciones, y sean utilizados tanto por los agentes sociales como por los propios trabajadores, se constata en el hecho de que hace más de 30 años Sterns y Doverspike (1989) ya propusieron cinco tipos de edad: edad cronológica, edad organizacional, edad funcional, edad subjetiva o psicosocial y edad de desarrollo a lo largo de la vida, aunque para la mayoría esta distinción no se aplica y ni siquiera es conocida. A partir de esta diferenciación en las conceptualizaciones de la edad, diversos investigadores (p. ej., De Lange et al., 2021; Kooij et al., 2008; Le Blanc et al., 2017) han indagado en sus implicaciones en la gestión de la edad y de la carrera media y tardía en contextos organizacionales.

En primer lugar, la edad cronológica obviamente se refiere a los años de vida transcurridos en un momento dado: es la edad que marca el calendario. Por su parte, la edad organizacional alude a los años de antigüedad en un puesto o en una organización, y suele definir la etapa o fase de carrera en que se encuentra el trabajador, así como la eventual obsolescencia de sus competencias en ese contexto (Kooij et al., 2008; Le Blanc et al., 2017). En tercer lugar, la edad funcional o basada en el desempeño, se refiere a la capacidad de un trabajador para realizar determinadas tareas laborales cotidianas, es decir, la relación entre los recursos disponibles y las demandas actuales del trabajo, así como a los efectos sobre su salud (Kooij et al., 2008). En relación con la edad funcional, el constructo más utilizado en la investigación es la capacidad de trabajo (work ability; Ilmarinen, 2009), tanto subjetiva o percibida como objetiva (Cadiz et al., 2019), y suele medirse en relación con los recursos y demandas laborales de tipo cognitivo y/o de tipo físico. Por su parte, la edad psicosocial o subjetiva alude a la percepción social y cultural de la edad, y se refiere a cómo se siente, se ve y actúa una persona, y con qué grupo de edad se identifica. Al mismo tiempo, también 
incluye las normas de edad en un contexto dado locupación, organización, sociedad) y los estereotipos de edad asociados (Le Blanc et al., 2017); por ejemplo, a qué edad debería jubilarse una persona, a qué edad ya no debería seguir desempeñando una profesión, qué significa tener determinada edad en un sector ocupacional u organización, etc. Por último, también incluye las decisiones que pueden tomarse cuando se etiqueta a un trabajador como mayor (Kooij et al., 2008). Y en quinto lugar, el concepto de edad vital se centra en los cambios intraindividuales debidos al transcurso de la persona por la edad adulta, y se relaciona con los cambios de comportamiento en cualquier momento del ciclo vital (Le Blanc et al., 2017); suele medirse por medio de la etapa vital en que se encuentra la persona o por su estatus familiar lestado civil o de convivencia, hijos dependientes, etc.), y por las identidades asociados a ellos (Kooij et al., 2008; Le Blanc et al., 2017).

A pesar de esta creciente evidencia y de la necesidad de utilizar diferentes enfoques sobre el significado de la edad, la realidad es que aún la mayor parte de los estudios empíricos (De Lange et al., 2021) siguen utilizando la edad cronológica como el principal indicador lincluso en muchas ocasiones, el únicol, además de utilizar también en su mayor parte diseños transversales que no permiten captar la complejidad del envejecimiento y su carácter multidimensional y dinámico. Y también las organizaciones e instituciones mantienen la edad cronológica como el único criterio para tomar decisiones acerca de la continuidad de la actividad laboral de los trabajadores a partir de ciertas edades, que cada vez son más tempranas. Un claro ejemplo de ello es el caso de la prejubilación, forzosa o voluntaria launque la voluntariedad de la decisión es habitualmente inexistente, a riesgo de empeorar las condiciones de trabajo en caso de no aceptarse), llevadas a cabo por las organizaciones de trabajadores a partir de los 50 años, tanto en países como España (p. ej., Alcover et al., 2012), como en otras fórmulas de jubilación forzosa en vigor en países de América Latina, si bien en la mayoría de estos países se establecen edades diferentes para hombres y mujeres (menores en cinco años, generalmente, para ellas).

En consecuencia, el primer reto para la investigación y para la práctica en ámbitos laborales y organizacionales es abandonar la edad cronológica como el principal criterio para percibir, valorar y tomar decisiones sobre los trabajadores mayores. La incorporación de los enfoques de edad alternativos mencionados puede ser una oportunidad para captar la complejidad de estos procesos y adaptar la gestión de los trabajadores mayores a su diversidad y valor real (Alcover et al., 2014). Así, promover la movilidad, tanto interna como externa, en las etapas medias y finales de la carrera laboral (Alcover \& Topa, 2018; Krekula, 2019) puede ser crucial para alcanzar el objetivo de mantener a los trabajadores mayores en el mercado laboral en buenas condiciones de salud y de desempeño laboral, y prolongar la vida laboral hasta más allá de las edades habituales de jubilación si las personas quieren y pueden trabajar.

\section{RETO/OPORTUNIDAD 2: UTILIZAR LA EVIDENCIA DISPONI- BLE SOBRE LA MOTIVACIÓN, LAS ACTITUDES Y EL DESEM- PEÑO DE LOS TRABAJADORES MAYORES}

Lo más importante de los cinco tipos de edad mencionados inicialmente definidos por Sterns y Doverspike (1989) es la gran variabilidad de estados relacionados con el trabajo que admiten. Personas con la misma edad cronológica pueden experimentar diferentes edades 
funcionales, subjetivas o psicosociales, organizacionales y vitales, y en consecuencia, mostrar competencias, actitudes, motivaciones, desempeños y niveles de salud enormemente dispares. La evidencia empírica muestra la existencia de una amplia variabilidad dentro de los grupos de edad en cuanto a los resultados del trabajo, y esa variabilidad tiende ser mayor a medida que las personas envejecen (Zacher et al., 2018). Al mismo tiempo, la variabilidad en capacidad, actitudes, desempeño y motivación puede relacionarse directamente con sus niveles de empleabilidad (De Lange et al., 2021). A continuación se analizan los resultados de la investigación sobre las relaciones entre la edad y las variables laborales más relevantes.

En primer lugar, hay evidencia de la relación nula entre la edad y el desempeño de tarea global ( $\mathrm{Ng} \&$ Feldman, 2008); así, la hipotética relación de U invertida entre la edad y el desempeño solo se confirma en trabajos de baja complejidad, encontrándose que la experiencia es más predictiva del desempeño laboral que la edad en trabajos de alta complejidad (Sturman, 2003). Estos datos indican que las demandas de desempeño se afrontan de manera que los trabajadores mayores movilizan todos los recursos personales disponibles, de modo que se compensan las posibles diferencias individuales entre trabajadores jóvenes y mayores ( $\mathrm{Ng} \&$ Feldman, 2008). También se muestra que los trabajadores de más edad tienden a realizar más comportamientos de ciudadanía organizacional, participan en menos comportamientos contraproducentes en el trabajo en general, y exhiben menos conductas agresivas en el lugar de trabajo, uso de sustancias (alcohol, tabaco, estimulantes, fármacos) durante la jornada laboral, y muestran menos retrasos y ausencias voluntarias -no debidas a enfermedades-, en particular ( $\mathrm{Ng}$ \& Feldman, 2008; véase Alcover, 2020, para un análisis más detallado).

En otro meta-análisis, Ng y Feldman (2010) estudiaron la relación entre la edad cronológica y 35 actitudes laborales clasificadas en tres grandes categorías: actitudes basadas en la tarea, actitudes basadas en la organización y actitudes relacionales o basadas en las personas. Los resultados mostraron que la edad cronológica estaba relacionada positivamente con la gran mayoría de las actitudes evaluadas (27 de las 35), lo que indica que las actitudes hacia el trabajo tienden a ser más positivas con la edad (Beier \& Kanfer, 2013), y que el arraigado estereotipo negativo no tenía ningún fundamento. En particular, Ng y Feldman (2010) comprobaron que existían diferencias significativas entre trabajadores jóvenes y mayores, en el sentido de que estos últimos muestran actitudes más favorables y/o menos desfavorables que los jóvenes. En síntesis (Alcover, 2020), las diferencias eran significativas en las actitudes basadas en las tareas (por ejemplo, mayores niveles de satisfacción general, de satisfacción con el trabajo en sí mismo y con el salario, e implicación con la tarea, así como menor ambigüedad, sobrecarga y conflicto de rol), en las actitudes relacionales respecto a los supervisores y compañeros (por ejemplo, mayor satisfacción con respecto a ambos, mayor confianza interpersonal, mayores percepciones de justicia interaccional, de calidad del intercambio líder-miembros en los equipos, y de apoyo de los supervisores y los compañeros), y en las actitudes basadas en la organización (por ejemplo, mayores niveles de compromiso afectivo, normativo y continuo, de identificación, de lealtad, de ajuste persona-organización, de justicia distributiva y procedimental, y de confianza organizacional, así como menores 
percepciones de inseguridad en el empleo y de ruptura del contrato psicológicol. No obstante, el estudio de $\mathrm{Ng}$ y Feldman (2010) identificaba algunas relaciones negativas entre la edad y los resultados relacionados con las tareas, como por ejemplo, la relación entre la edad y la satisfacción con las oportunidades de promoción, y la relación entre la edad y la sobrecarga de trabajo. Estos efectos negativos seguían siendo significativos (aunque atenuados) tras controlar la antigüedad en la organización. En suma, como subrayaban Beier y Kanfer (2013), estos resultados sugieren que los trabajadores mayores están relativamente satisfechos con la mayoría de los aspectos de su trabajo, pero que se sienten algo sobrepasados por las demandas y las cargas de trabajo y también limitados en sus oportunidades de crecimiento y desarrollo profesional. Este último aspecto es importante, ya que existe relación entre la dimensión oportunidades restantes en la carrera, relacionada con la perspectiva de tiempo futuro, y la empleabilidad percibida en función de la edad (Froehlich et al., 2016).

En un tercer meta-análisis realizado por estos autores (Ng \& Feldman, 2012), los resultados permitieron comprobar que los estereotipos más comunes acerca de los trabajadores mayores (es decir, que están menos motivados, menos dispuestos a participar en programas de formación y en el desarrollo de carrera, más resistentes y menos dispuestos a los cambios, más desconfiados, con peor salud y más vulnerables a los desequilibrios entre la vida laboral y la familiar) no encontraban en general apoyo en los resultados de los estudios empíricos. El único estereotipo que encuentra evidencia empírica es el relativo a la menor disposición para participar en programas de formación y en el desarrollo de carrera; los otros cinco estereotipos no tienen ninguna base, lo que demuestra de nuevo la importancia de modificar las percepciones sesgadas y las opiniones preconcebidas acerca de los trabajadores mayores en función de su edad cronológica (Alcover, 2020). A pesar de esta evidencia, en la práctica los estereotipos de edad acerca del desempeño esperado siguen influyendo en las decisiones de contratación, y es uno de los principales motivos de discriminación por edad en el trabajo (Petery et al., 2020). En definitiva, según Beier y Kanfer (2013), los resultados de varios estudios meta-analíticos en este ámbito sugieren que es esperable que los trabajadores de más edad realicen las tareas laborales al mismo nivel que los más jóvenes, y contribuyan positivamente al logro de los resultados organizacionales sin diferencias significativas respecto a éstos.

En suma, el segundo reto para la investigación y para la práctica en ámbitos laborales y organizacionales se centra en modificar los estereotipos negativos asociados a la edad de empleadores, gerentes, trabajadores y agentes sociales, estereotipos que afectan a sus percepciones, valoraciones y decisiones relativas a sus competencias, sus actitudes, su motivación, su desempeño, su salud y otros factores relacionados con los resultados del trabajo. Este cambio de estereotipos facilitará la reducción de los prejuicios de edad y, en última instancia, de las conductas y prácticas de discriminación por razones de edad (ageism), cuyo diagnóstico y prevención resultan claves para garantizar los derechos laborales de los adultos mayores en los diferentes países (Alcover, Nazar et al., 2021). Con ello, también se logrará reducir los potenciales efectos de la discriminación por edad en el bienestar y la salud ocupaciones de los adultos mayores. 


\section{RETO/OPORTUNIDAD 3: HACIA LA EMPLEABILIDAD SOS- TENIBLE DE LOS TRABAJADORES MAYORES}

Como se ha comprobado en los análisis de los Retos/ Oportunidades 1 y 2 , en el actual contexto de reformulación de lo que significa ser un "trabajador mayor" y los estereotipos asociados a esta categoría, ha cobrado interés la identificación de los factores antecedentes y concurrentes -tanto individuales como contextualesque pueden facilitar y aumentar su empleabilidad y la sostenibilidad de sus carreras a lo largo del tiempo (Van der Heijden et al., 2020). Como consecuencia de ello, términos como sostenibilidad de la carrera profesional, empleabilidad de los trabajadores de edad avanzada o empleabilidad sostenible se han hecho frecuentes en la investigación y la práctica en este ámbito, al mismo tiempo que se sugiere abandonar la edad cronológica para evaluar a los trabajadores y su empleabilidad en estas etapas de su carrera (De Lange et al., 2021).

Aunque los constructos de 'sostenibilidad' y 'empleabilidad' cuentan con una larga tradición de investigación y aplicación en diversas disciplinas, desde la economía y la psicología hasta la ecología, la combinación de ambos en un único constructo es más reciente, apenas una década desde que se inició la investigación en países europeos, especialmente en Países Bajos. La integración de ambos en el término empleabilidad sostenible intenta captar los elementos dinámicos que influyen en la capacidad de una persona para mantener de forma sostenible la empleabilidad, la salud y el bienestar a lo largo de su vida laboral (Alcover, Mazzetti, et al., 2021). Como resultado, el constructo comprende tres componentes principales: empleabilidad, sostenibilidad y tiempo (Fleuren et al., 2020).
Si bien no existe una única definición aceptada por los investigadores en esta área, la revisión de la literatura (Alcover, Mazzetti, et al., 2021; Fleuren et al. 2020) permite identificar dos enfoques principales. Por una parte, tomando como base el enfoque de 'capability' del economista Amartya Sen, van der Klink et al. (2016) consideran que la empleabilidad sostenible se refiere a que los trabajadores pueden alcanzar oportunidades tangibles en forma de un conjunto de capacidades [capability set for work] a lo largo de su vida laboral. Y también comprende el disfrute de unas condiciones laborales que les permiten realizar con su trabajo contribuciones, tanto actualmente como en el futuro, manteniendo niveles adecuados de salud y bienestar. Para ello, los trabajadores han de disponer, por una parte, de un contexto laboral que se lo facilite y, por otra, de la actitud y la motivación para aprovechar las oportunidades del contexto (van der Klink et al., 2016).

Como se puede apreciar en esta definición, su enfoque requiere, para lograr la empleabilidad sostenible, de la disponibilidad de condiciones personales y contextuales (laborales y organizacionales) que "permitan a los trabajadores convertir sus aportaciones personales y laborales en estas capacidades loportunidades reales de funcionamiento laboral valioso)" (van der Klink et al., 2016, p. 74). Con objetivo de operacionalizar su medida, los autores han elaborado un instrumento específico (Abma et al., 2016) que pretende medir siete aspectos de la capacidad para el trabajo: (a) utilizar los conocimientos y las competencias; (b) desarrollar los conocimientos y las competencias; (c) participar en las decisiones importantes; (d) establecer contactos significativos en el trabajo; (e) fijar los propios objetivos; (f) tener buenos ingresos; y $(\mathrm{g})$ contribuir a algo valioso; 
además, incluye una medida de un solo valoración de la capacidad general. Esta valoración es auto-percibida, es decir, se mide la empleabilidad sostenible percibida.

Y por otra parte, el segundo enfoque (Fleuren et al., $2018,2020)$ considera la empleabilidad sostenible como un constructo de segundo orden, compuesto por nueve factores de primer orden, que captan el componente de 'empleabilidad' del constructo (Fleuren et al., 2020), los cuales poseen una naturaleza inherentemente longitudinal. Estos factores de primer orden son: (a) la salud subjetiva; (b) la necesidad de recuperación; (c) la fatiga; (d) la capacidad de trabajo; (e) la brecha de habilidades; (f) el rendimiento; (g) la empleabilidad; (h) la motivación; y (i) la satisfacción laboral (Fleuren et al., 2018). Además, para captar el componente de 'sostenibilidad', la definición debe incluir los incrementos o al menos la estabilidad de la 'empleabilidad' a lo largo del tiempo, es decir, incluir la relación de "los posibles predictores con la pendiente de la 'empleabilidad', [lo que] permite estimar sus efectos sobre la sostenibilidad de la empleabilidad', o mejor dicho, sobre la empleabilidad sostenible “ (Fleuren et al., 2020, p. 15).

A partir de este fundamento, la definición más reciente propuesta por los autores es la siguiente:

La empleabilidad sostenible significa que la capacidad de un individuo para funcionar en el trabajo y en el mercado laboral, o su 'empleabilidad', no se ve afectada negativamente, y preferiblemente lo es positivamente, por su utilización por parte de ese individuo a lo largo del tiempo. Esta capacidad puede captarse de forma significativa como una combinación de nueve indicadores les decir, el estado de salud percibido, la capacidad de trabajo, la necesidad de recuperación, la fatiga, la satisfacción en el trabajo, la motivación para trabajar, la empleabilidad percibida, la brecha de habilidades y el rendimiento en el trabajo) que describen conjuntamente lo bien que un individuo puede estar empleado en diferentes momentos de su vida laboral. (Fleuren et al., 2020, p. 15)

Además de estas dos orientaciones generales, en la investigación también se han utilizado otras operacionalizaciones ad hoc, por lo que la integración de los resultados obtenidos hasta ahora resulta limitada debido a la disparidad de medidas y de criterios o indicadores de la empleabilidad sostenible utilizados, como ha puesto de manifiesto una revisión reciente (Alcover, Mazzetti, at al. 2021). No obstante, el concepto de empleabilidad sostenible cuenta con grandes potencialidades para evaluar a lo largo del tiempo la capacidad de trabajo de los adultos mayores (sin excluir a los trabajadores de otros grupos de edad, puesto que la sostenibilidad de la capacidad laboral se extiende durante toda la carrera o vida laboral), de modo que su desarrollo futuro podrá ser muy útil en este campo. Un aspecto que recientemente se ha incorporado al análisis es el riesgo que representan para la empleabilidad sostenible de los trabajadores mayores los impactos en los puestos de trabajo y las ocupaciones de la robótica, la inteligencia artificial, la automatización y, en general, la digitalización (Alcover, Guglielmi et al., 2021). Estas transformaciones tecnológicas pueden aumentar la vulnerabilidad de este grupo de edad (por la doble vulnerabilidad derivada de la edad y la tecnología) y afectar negativamente al mantenimiento del empleo provocando decisiones, forzadas o voluntarias, de retiro temprano, acortando las carreras laborales y abocando, en el caso de los países iberoamericanos, a la salida del mercado formal y la entrada en el informal. Como ocurre con otras formas de vulnerabilidad laboral, las consecuencias se pueden traducir en mayor probabi- 
lidad de sufrir bullying y, consecuentemente, elevados niveles de estrés, afectando de manera severa sobre la salud mental de los trabajadores y, especialmente, de las mujeres trabajadoras (Ansoleaga et al., 2019).

En el ámbito específico de los países iberoamericanos, uno de los retos para la aplicación del constructo de empleabilidad sostenible se encuentra en el elevado volumen de trabajo informal. Según un reciente informe elaborado por la CEPAL IComisión Económica para América Latina y el Caribe, organismo dependiente de la Organización de las Naciones Unidas) y la OIT IOrganización Internacional del Trabajo; CEPAL/OIT, 2019), se han identificado en los países de América Latina y el Caribe tres indicadores de nuevos retrocesos en el cumplimiento del Objetivo de Desarrollo Sostenible (ODS) No 8 de la Agenda 2030, que busca promover el crecimiento económico sostenido, inclusivo y sostenible, el empleo pleno y productivo y el trabajo decente para todos. Los tres indicadores detectados se refieren a (1) el aumento significativo del trabajo por cuenta propia, en detrimento del trabajo asalariado; (2) el surgimiento y rápido crecimiento de nuevas formas de trabajo intermediadas por plataformas digitales; y (3), al menos en varios países, una mayor informalidad del empleo asalariado, debido tanto a la debilidad en la creación de este tipo de empleo, como al aumento de la informalización de empleos existentes como consecuencia de estrategias organizacionales definidas por el objetivo de la reducción de costos (CEPAL/OIT, 2019). En definitiva, el aumento de la informalidad del trabajo, tanto la estructural como la sobrevenida, y el crecimiento de nuevas formas de trabajo que, habitualmente, se desarrollan fuera de las regulaciones existentes, provocan un incremento de trabajadores que no disfru- tan de los derechos laborales y sociales establecidos por las legislaciones nacionales y alejan a la región del cumplimiento del Objetivo $N^{0} 8$ de Desarrollo Sostenible [CEPAL/OIT, 2019]. Además, las consecuencias derivadas de la pandemia de la COVID-19 han acentuado las brechas estructurales de los mercados de trabajo en América Latina, como muestra el reciente informe de Panorama Social de CEPAL (2021).

Relacionado con lo anterior, la investigación reciente también se ha ocupado de las carreras sostenibles (De Vos et al., 2020), un término que cuenta con un enfoque sistémico y dinámico para analizar los factores y los cambios que afectan a la sostenibilidad de las carreras a lo largo del tiempo. Respecto al primer enfoque, si bien la carrera profesional de cada persona es individual, se encuentra interconectada tanto con los contextos en que se produce como con otros actores implicados, al tiempo que es influida por ellos. Según De Vos et al. (2020),

el reto de la sostenibilidad va más allá de la gestión individual de la carrera y requiere la participación activa de todas las partes implicadas, como la familia y los compañeros del individuo, el supervisor o los supervisores, el empleador, el sistema educativo y la sociedad. (p. 2)

En cuanto al enfoque dinámico, las carreras son procesos donde los factores de la persona y los de su contexto cambian a lo largo del tiempo, afectando a la sostenibilidad de las carreras. En consecuencia, las carreras sostenibles se conciben como un proceso cíclico de autorregulación en el que las experiencias y los acontecimientos, tanto positivos como negativos, y la percepción e interpretación de la persona y las diferentes partes implicadas en el proceso, generan oportunidades para 
un "aprendizaje dinámico" en el contexto de carrera (De Vos et al., 2020). De nuevo aquí, las oportunidades para la sostenibilidad de las carreras en países iberoamericanos se enfrenta a las dificultades generadas por la elevada informalidad del empleo y la precariedad e incertidumbre de las trayectorias laborales (MaderoCabib et al., 2020). En este sentido, un reciente estudio realizado en Chile (Yeves et al., 2019) muestra como en el caso de los trabajadores mayores su percepción de empleabilidad no reducía las consecuencias negativas de experimentar inseguridad en el empleo, lo que puede ser un indicador claro de la vulnerabilidad y la baja sostenibilidad de carrera experimentadas incluso por quienes perciben alta empleabilidad, afectando secundariamente a su bienestar y salud ocupacional.

En definitiva, el tercer reto para la investigación y para la práctica en ámbitos laborales y organizacionales se centra en cómo contrarrestar los factores estructurales del mercado de trabajo en Iberoamérica mencionados y los vectores de vulnerabilidad, inseguridad, incertidumbre y precariedad que generan amenazando la empleabilidad y la sostenibilidad de las carreras a lo largo del tiempo, especialmente, la de los adultos mayores.

\section{CONCLUSIONES Y FUTURAS LÍNEAS DE INVESTIGACIÓN E INTERVENCIÓN}

El análisis de estos tres grandes ejes de retos y oportunidades para los trabajadores y las organizaciones relacionados con la edad, la actividad laboral y la salud ocupacional permite identificar los principales factores a considerar en la gestión de los trabajadores mayores, así como en su bienestar y salud. Como recomendación general, es necesario transmitir desde la investigación realizada en el ámbito de la Psicología de las Organizaciones y el Trabajo a los responsables y gerentes de las empresas e instituciones, que el tradicional enfoque de la "depreciación" aplicado al envejecimiento no tiene fundamento empírico. Esta tradicional perspectiva basada en la "depreciación” (Stein \& Rocco, 2001) considera que las personas están más motivadas y realizan sus mayores aportaciones al trabajo en las etapas iniciales y medias de su carrera, declinando a medida que maduran y envejecen. La edad en que se puede manifestar el deterioro y la obsolescencia es variable, pero este perspectiva considera que el "trabajador mayor" es un trabajador con "déficits", con "problemas", en cierto modo, cada vez menos "útil". Esta categorización refuerza los estereotipos negativos, dificulta las relaciones intergeneracionales en el trabajo, favorece el desarrollo de prejuicios hacia los adultos mayores, desencadena conductas y decisiones de discriminación laboral y potencia su abandono temprano del mercado laboral (Alcover, 2020; Topa \& Alcover, 2015; Topa et al., 2018).

Como señalaron Stein y Rocco (2001), esta perspectiva se asienta en varios mitos, que la evidencia empírica actual no respalda: los mitos ya descritos de la edad cronológica, de la productividad en declive y de la pérdida de motivación; el mito de la carrera profesional, que considera que las organizaciones no deberían invertir en la formación y el desarrollo de la carrera de los trabajadores mayores debido al bajo retorno que se espera obtener de la inversión que se hiciera en ellos, y, por último, el mito de la jubilación, derivado de esta visión de pendiente descendente que solo puede conducir al retiro. Sin embargo, esta visión todavía predominante tanto entre los responsables organizacionales, como en los propios trabajadores y en el imaginario social, tiene 
un contrapeso en la perspectiva de la "conservación" (Kooij \& Van de Voorde, 2015; Pak et al., 2019), que plantea que las organizaciones y las personas pueden llevar a cabo conjuntamente acciones e intervenciones para mantener e incluso desarrollar las competencias, la motivación y la capacidad de trabajo de los trabajadores en sus etapas medias y finales de la carrera laboral (Wang et al., 2013). Precisamente una gestión de personas adaptada a la situación real de ese grupo de edad heterogéneo formado por los trabajadores mayores puede servir para alcanzar los objetivos de la empleabilidad y la carrera sostenible analizados anteriormente. Las futuras investigaciones e intervenciones deben partir de estos supuesto basados en la evidencia.

En un trabajo anterior (Alcover, 2019), y con el objetivo de dar respuesta al reto de la gestión de la edad en las organizaciones, se formuló un modelo integrado de gestión de personas basado en la evidencia dirigido a los trabajadores mayores, que incluía una perspectiva de la evolución temporal y las trayectorias en las etapas medias y finales de la carrera y las correspondientes prácticas de gestión de personas que serían más adecuadas y eficaces a corto, medio y largo plazo. Esa propuesta presentaba un enfoque alejado de la utilización exclusiva de las prácticas universalistas o generales de la gestión de personas, pero también lejos de las prácticas "edadistas", y por lo tanto, reificadoras de una categoría artificial (trabajador mayor) y carente de entidad epistémica, aplicadas solo en función de criterios cronológicos que no reflejan el estado, las motivaciones y las necesidades reales de las personas. Como ya se mencionó, el envejecimiento no es un proceso uniforme, y sus consecuencias físicas, cognitivas, emocionales y sociales presentan una gran variabilidad que las prác- ticas de gestión de personas deben considerar para garantizar su eficacia. También es necesario realizar una previsión de las trayectorias y la evolución temporal de las etapas medias y finales de la carrera profesional y de las correspondientes prácticas de gestión de personas que serían más adecuadas a corto, medio y largo plazo (Alcover, 2019). Esta perspectiva temporal y dinámica es necesaria en base a la evidencia acumulada desde la perspectiva del ciclo vital sobre el proceso de envejecimiento, y se alinea con los planteamientos dirigidos a facilitar la sostenibilidad del trabajo a lo largo de la vida analizados más arriba (De Lange et al., 2015; Fleuren et al, 2018, 2020; van der Klink et al., 2016). También aquí, tanto la investigación como la gestión y las intervenciones organizacionales han de aprovechar los conocimientos acumulados para continuar avanzando en la mejora de la empleabilidad, el bienestar y la salud laboral de los trabajadores mayores.

En resumen, para afrontar los retos de la edad en el trabajo y convertirlos en oportunidades es necesario: (1) cambiar los estereotipos y reducir los prejuicios acerca de lo que significa ser un "trabajador mayor"; (2) concienciar a las organizaciones para que desarrollen un enfoque estratégico de gestión de una fuerza de trabajo intergeneracional; (3) prevenir y evitar la discriminación laboral por edad; (4) afrontar de manera realista los cambios (positivos y negativos) en las capacidades (cognitivas, físicas, emocionales y relacionales) y en la salud asociados al envejecimiento; (5) desarrollar sistemas de gestión de personas adaptados a las necesidades de cada momento de la carrera profesional; (6) desarrollar y gestionar la carrera profesional de las etapas media y finales y facilitar políticas y prácticas para prolongar la vida laboral; (7) gestionar las transiciones hacia 
el retiro; (8) tomar conciencia de que la gestión de la edad en las organizaciones no es una opción, sino una necesidad; (9) crear sistemas de protección para los trabajadores mayores que deben seguir trabajando por motivos económicos; y (10) prestar especial atención a los riesgos asociados al empleo y al trabajo en el sector informal, que no cuentan con suficientes mecanismos de protección social ni de la salud.

\section{Declaración de conflicto de interés}

Los autores no declaran ningún conflicto de interés.

\section{REFERENCIAS}

Abma, F. I., Brouwer, S., de Vries, H. J., Arends, I., Robroek, S. J., Cuijpers, M. P., van der Wilt, G. J., Bültmann, U., \& van der Klink, J. J. (2016). The capability set for work: development and validation of a new questionnaire. Scandinavian Journal of Work, Environment \& Health, 42(1), 34-42. https://doi.org/10.5271/sjweh.3532

Aburto, J. M., Schöley, J., Kashnitsky, I., Zhang, L., Rahal, C., Missov, T. I., Mills, M. C., Dowd, J. B., \& Kashyap, R. (2021). Quantifying impacts of the COVID-19 pandemic through lifeexpectancy losses: a population-level study of 29 countries. International Journal of Epidemiology, dyab207. Advance online publication. https://doi.org/10.1093/ije/dyab207

Alcover, C. M. (2019). Cómo afrontar el desafío de la gestión de recursos humanos de los trabajadores mayores: un modelo integrado basado en la evidencia. Revista de Trabajo y Seguridad Social, CEF, 437-438, 203-234.

Alcover, C. M. (2020). Empleo sostenible: Edad, trabajo y alternativas al retiro en la sociedad 4.0. Ediciones Díaz de Santos.

Alcover, C. M., Crego, A., Guglielmi, D., \& Chiesa, R. (2012). Comparison between the Spanish and Italian early work retirement models: A cluster analysis approach. Personnel Review, 41(3), 380-403. https://doi.org/10.1108/00483481211212995

Alcover, C.-M., Guglielmi, D., Depolo, M., \& Mazzetti, G. (2021). “Aging-and-Tech Job Vulnerability": A proposed framework on the dual impact of aging and Al, robotics, and automation among older workers. Organizational Psychology Review, 11(2), 175-201. https://doi.org/10.1177/2041386621992105

Alcover, C. M., Mazzetti, G., \& Vignoli, M. (2021). Sustainable Employability in the Mid and Late Career: An Integrative Review. Journal of Work and Organizational Psychology. Ahead of print. https://doi.org/10.5093/jwop2021a16

Alcover, C. M., Nazar, G., Bargsted, M., Ramírez-Vielma, R., Pulido, N., \& Rodríguez, L. (2021). Transcultural validation of the Nordic Age Discrimination Scale for the Spanish-speaking working populations. Spanish Journal of Psychology, under review. 
Alcover, C. M., \& Topa, G. (2018). Work characteristics, motivational orientations, psychological work ability and job mobility intentions of older workers. PLOS ONE, 13(4), Article e0195973. https://doi.org/10.1371/journal.pone.0195973

Alcover, C. M., Topa, G., \& Fernández, J. J. (2014). La gestión organizacional de los trabajadores mayores y los procesos de mantenimiento, prolongación y salida de la vida laboral [The organizational management of older workers and processes of maintaining, extending and leaving employment]. Papeles del Psicólogo, 35(2), 91-98.

Alcover, C. M., Topa, G., Parry, E., Fraccaroli, F., \& Depolo, M. (eds.) (2014). Bridge Employment: A Research Handbook. Routledge.

Ansoleaga, E., Ahumada, M., \& González-Santa Cruz, A. (2019). Association of Workplace Bullying and Workplace Vulnerability in the Psychological Distress of Chilean Workers. International Journal of Environmental Research and Public Health, 16(20), 4039. https://doi.org/10.3390

Avdeev, A., Eremenko, T., Festy, P., Gaymu, J., Le Bouteillec, N. \& Springe, S. (2011). Populations et tendances démographiques des pays européens (1980-2010). Population, 66, 9-133. https://doi.org/10.2307/41441033

Baltes, P. B. (1987). Theoretical propositions of life-span developmental psychology: On the dynamics between growth and decline. Developmental Psychology, 23(5), 611-626. https://doi.org/10.1037/0012-1649.23.5.611

Bauer, J. M., \& Sousa-Poza, A. (2015). Impacts of informal caregiving on caregiver employment, health, and family. Journal of Population Ageing, 8(3), 113-145. https://doi.org/10.1007/s12062-015-9116-0

Beier, M. E., \& Kanfer, R. (2013). Work performance and the older worker. En J. Field, R. J. Burke, \& C. L. Cooper (eds.), The SAGE Handbook of Aging, Work and Society (pp. 97-117). SAGE Publications Ltd, https://www.doi.org/10.4135/9781446269916.n6

Berganza, J. C., Campos, R., Martínez Casillas, E., \& Pérez, J. (2020). El agotamiento del dividendo demográfico en Latinoamérica: retos para las políticas económicas y sociales. Boletín Económico, Banco de España, 1/2020, 1-14.
Boehs, S. d. T. M., Medina, P. F., Bardagi, M. P., Luna, I. N., \& Silva, N. (2017). Revisão da literatura Latino-Americana sobre aposentadoria e trabalho: Perspectivas psicológicas [Review of Latin American literature on retirement and work: Psychological perspectives]. Revista Psicologia Organizações e Trabalho, 17(1), 54-61. https://doi.org/10.17652/rpot/2017.1.11598

Burch, K. A., Dugan, A. G., \& Barnes-Farrell, J. L. (2019). Understanding What Elder-care Means for Employees and Organizations: A Review and Recommendations for Future Research. Work, Aging and Retirement, 5(1), 44-72. https://doi.org/10.1093/workar/way011

Burke, R. J. (2017). The sandwich generation: individual, family, organizational and societal challenges and opportunities. In R. J. Burke \& L. M. Calvano (eds.), The Sandwich Generation. Caring for Oneself and Others at Home and at Work (pp. 3-39). Edward Elgar.

Cadiz, D. M., Brady, G., Rineer, J. R. \& Truxillo, D. M. (2019). A Review and Synthesis of the Work Ability Literature. Work, Aging and Retirement, 5(1), 114138. https://doi.org/10.1093/workar/way010

Carstensen, L. L., Isaacowitz, D. M., \& Charles, S. T. (1999). Taking time seriously. A theory of socioemotional selectivity. The American psychologist, 54(3), 165-181. https://doi.org/10.1037//0003-066x.54.3.165

CEPAL (2021). Panorama Social de América Latina 2020. Naciones Unidas.

CEPAL/OIT 12019. Coyuntura Laboral en América Latina y el Caribe. El futuro del trabajo en América Latina y el Caribe: antiguas y nuevas formas de empleo y los desafíos para la regulación laboral. Naciones Unidas.

Converso, D., Sottimano, I., Viotti, S., \& Guidetti, G. (2020). I'll be a caregiver-employee: Aging of the workforce and family-to-work conflicts. Frontiers in Psychology, 11:246. https://doi.org/10.3389/fpsyg.2020.00246

Crawford, J. O., Graveling, R. A., Cowie, H. A., \& Dixon, K. (2010). The health safety and health promotion needs of older workers. Occupational Medicine, 60(3), 184-192. https://doi.org/10.1093/occmed/kqq028 
De Lange, A. H., Kooij, D. T. A. M., \& Van der Heijden, B. I. J. M. (2015). Human Resource Management and sustainability at work across the lifespan: An integrative perspective. En L. M. Finkelstein et al. (Eds.), Facing the Challenges of a Multi-Age Workforce. A Use-Inspired Approach (pp. 50-79). Routledge.

De Lange, A. H., Van der Heijden, B., Van Vuuren, T., Furunes, T., De Lange, C., \& Dikkers, J. (2021). Employable as We Age? A Systematic Review of Relationships Between Age Conceptualizations and Employability. Frontiers in Psychology, 11, 605684. https://doi.org/10.3389/fpsyg.2020.605684

De Souza, L. R., Queiroz, B.L., \& Skirbekk, V. F. (2019). Trends in health and retirement in Latin America: Are older workers healthy enough to extend their working lives? The Journal of the Economics of Ageing, 13(C), 72-83. https://doi.org/10.1016/j.jeoa.2018.03.008

De Vos, A., Van der Heijden, B. I. J. M., \& Akkermans, J. (2020). Sustainable careers: Towards a conceptual model. Journal of Vocational Behavior, 117, Article 103196. https://doi.org/10.1016/j.jvb.2018.06.011

Fernández, F., Nazar, G., \& Alcover, C. M. (2018). Modelo de envejecimiento activo: causas, indicadores y predictores en adultos mayores en Chile. Acción Psicológica, 15(2), 109-128. https://doi.org/10.5944/ap.15.2.22903

Fleuren, B. P. I., de Grip, A., Jansen, N. W. H., Kant, I., \& Zijlstra, F.R.H. (2020). Unshrouding the Sphere from the Clouds: Towards a Comprehensive Conceptual Framework for Sustainable Employability. Sustainability, 12, 6366. https://doi.org/10.3390/su12166366

Fleuren, B., van Amelsvoort, L., Zijlstra, F., de Grip, A., \& Kant, I. (2018). Handling the reflective-formative measurement conundrum: A practical illustration based on sustainable employability. Journal of Clinical Epidemiology, 103, 71-81. https://doi.org/10.1016/j.jclinepi.2018.07.007

Froehlich, D. E., Beausaert, S., \& Segers, M. (2016). Aging and the motivation to stay employable. Journal of Managerial Psychology, 31(3), 756-770. https:// doi.org/10.1108/JMP-08-2014-0224
Gietel-Basten, S., Saucedo, S., \& Scherbov, S. (2020). Prospective measures of aging for Central and South America. PloS ONE, 15(7), e0236280. https:// doi.org/10.1371/journal.pone.0236280

Henle, C. A., Fisher, G. G., McCarthy, J. Prince, M. A., Mattingly, V. P. \& Clancy, R. L. (2020). Eldercare and childcare: How does caregiving responsibility affect job discrimination? Journal of Business and Psychology, 35(4), 59-83. https://doi.org/10.1007/s10869-019-09618-x

Ilmarinen J. (2009). Work ability--a comprehensive concept for occupational health research and prevention. Scandinavian Journal of Work, Environment \& Health, 35(1), 1-5. https://doi.org/10.5271/sjweh.1304

Kooij, D., de Lange, A., Jansen, P., \& Dikkers, J. (2008). Older workers' motivation to continue to work: Five meanings of age: A conceptual review. Journal of Managerial Psychology, 23(4), 364-394. https://doi.org/10.1108/02683940810869015

Kooij, D. T. A. M. \& Van de Voorde, K. (2015). Strategic HRM for older workers. En P. M. Bal, D. T. A. M. Kooij, \& D. M. Rousseau (Eds.), Aging Workers and the EmployeeEmployer Relationship (pp. 57-72). Springer.

Kooij, D., Zacher, H., Wang, M., \& Heckhausen, J. (2020). Successful aging at work: A process model to guide future research and practice. Industrial and Organizational Psychology, 13(3), 345-365. https://doi.org/10.1017/iop.2020.1

Krekula, C. (2019). Time, precarisation and age normality: on internal job mobility among men in manual work. Ageing \& Society, 39(10), 2290-2307. https:// doi.org/10.1017/S0144686X1800137X

Le Blanc, P. M., Van der Heijden, B., \& Van Vuuren, T. (2017). "I WILL SURVIVE" A Construct Validation Study on the Measurement of Sustainable Employability Using Different Age Conceptualizations. Frontiers in Psychology, 8, 1690. https://doi.org/10.3389/fpsyg.2017.01690

Leeson, G. (2013) The demographics of ageing in Latin America, the Caribbean and the Iberian Peninsula, 1950-2050. En V. Montes de Oca (ed.) Envejecimiento en America Latina y el Caribe (pp. 53-71). UNAM. 
Madero-Cabib, I., Biehl, A., Sehnbruch, K., Calvo, E., \& Bertranou, F. (2019). Private Pension Systems Built on Precarious Foundations: A Cohort Study of LaborForce Trajectories in Chile. Research on Aging, 41(10), 961-987. https://doi.org/10.1177/0164027519874687

Moulaert, T. \& Biggs, S. (2013). International and European policy on work and retirement: Reinventing critical perspectives on active ageing and mature subjectivity. Human Relations, 66(1), 23-43. https://doi.org/10.1177/0018726711435180

Ng, T. W. H., \& Feldman, D. C. (2008). The relationship of age to ten dimensions of job performance. Journal of Applied Psychology, 93(2), 392-423. https://doi.org/10.1037/0021-9010.93.2.392

Ng, T. W. H., \& Feldman, D. C. (2010). The relationships of age with job attitudes: A meta-analysis. Personnel Psychology, 63(3), 677-718. https://doi.org/10.1111/j.1744-6570.2010.01184.x

Ng, T. W. H., \& Feldman, D. C. (2012). Evaluating six common stereotypes about older workers with meta-analytical data. Personnel Psychology, 65(4), 821-858. https://doi.org/10.1111/peps.12003

Osborne, K., \& Newman, L. (2012). Active Ageing and Employment: Summary of the research evidence. Flinders Universtiy.

Pak, K., Kooij, D. T. A. M., De Lange, A. H., \& Van Veldhoven, M. J. P. M. (2019). Human Resource Management and the ability, motivation and opportunity to continue working: A review of quantitative studies. Human Resource Management Review, 29(3), 336-352. https://doi.org/10.1016/j.hrmr.2018.07.002

Petery, G. A., Wee, S., Dunlop, P. D., \& Parker, S. K. (2020). Older workers and poor performance: Examining the association of age stereotypes with expected work performance quality. International Journal of Selection and Assessment, 28(4), 510-521. https://doi.org/10.1111/ijsa.12309

Population Reference Bureau (2021). International Data - World Latinoamerica and the Caribbean. https://www.prb.org/ international/geography/latin-america-and-the-caribbean/

Sanderson, W. C. \& Scherbov, S. (2010). Remeasuring aging. Science, 329, 1287-1288. https://doi.org/10.1126/science.1193647
Sanderson, W. C., Scherbov, S., \& Gerland, P. (2017). Probabilistic population aging. PloS ONE, 12(6), e0179171. https://doi.org/10.1371/journal.pone.0179171

Sturman, M. C. (2003). Searching for the Inverted U-Shaped Relationship Between Time and Performance: MetaAnalyses of the Experience/Performance, Tenure/ Performance, and Age/Performance Relationships. Journal of Management, 29(5), 609-640. https:// doi.org/10.1016/S0149-2063(03)00028-X

Topa, G., \& Alcover, C.-M. (2015). Psychosocial factors in retirement intentions and adjustment: A multi-sample study. The Career Development International, 20(4), 384-408. https://doi.org/10.1108/CDI-09-2014-0129

Topa, G., Alcover, C. M., Moriano, J. A., \& Depolo, M. (2014). Bridge employment quality and its impact on retirement adjustment: A structural equation model with SHARE panel data. Economic and Industrial Democracy, 35(2), 225-244. https://doi.org/10.1177/0143831X12475242

Topa, G., Depolo, M., \& Alcover, C. M. (2018). Early Retirement: A Meta-Analysis of Its Antecedent and Subsequent Correlates. Frontiers in psychology, 8, 2157. https://doi.org/10.3389/fpsyg.2017.02157

Troncoso Pantoja, C., Díaz Martínez, X., Martorell Pons, M., Nazar Carter, G., Concha Cisternas, Y., \& Cigarroa Cuevas, I. (2020). Revisión de estrategias para promover el envejecimiento activo en Iberoamérica. MediSur, 18(5),899-906.

Truxillo, D. M., Cadiz, D. M., \& Hammer, L. B. (2015). Supporting the aging workforce: A review and recommendations for workplace intervention research. Annual Review of Organizational Psychology and Organizational Behavior, 2, 351-381. https://doi. org/10.1146/annurev-orgpsych-032414-111435

United Nations (2019). World Population Prospects 2019: Highlights. DESA, Population Division. https:// population.un.org/wpp/Publications/

Van der Heijden, B., De Vos, A., Akkermans, J., Spurk, D., Semeijn, J., Van der Velde, M., \& Fugate, M. (2020). Sustainable careers across the lifespan: Moving the field forward. Journal of Vocational Behavior, 117, 103344. https://doi.org/10.1016/j.jvb.2019.103344 
Vives, A., Gray, N., González, F., \& Molina, A. (2018). Gender and Ageing at Work in Chile: Employment, Working Conditions, Work-Life Balance and Health of Men and Women in an Ageing Workforce. Annals of Work Exposures and Health, 62(4), 475-489. https://doi.org/10.1093/annweh/wxy021

Wang, M., Olson, D. A., \& Shultz, K. S. (2013). Mid and Late Career. An Integrative Perspective. Routledge.

Yeves, J., Bargsted, M., Cortes, L., Merino, C., \& Cavada, G. (2019). Age and Perceived Employability as Moderators of Job Insecurity and Job Satisfaction: A Moderated Moderation Model. Frontiers in Psychology, 10: 799. https://doi.org/10.3389/fpsyg.2019.00799

Zacher, H., Kooij, D. A. T. M. \& Beier, M. E. (2018). Active aging at work: Contributing factors and implications for organizations. Organizational Dynamics, 37, 47-55. https://doi.org/10.1016/j.orgdyn.2017.08.001 